\title{
Chimeric antigen receptor-natural killer cells: Novel insight into immunotherapy for solid tumors (Review)
}

\author{
XIAOBO PENG*, LING CHEN*, LONGPEI CHEN*, BIN WANG, YIRAN WANG and XIANBAO ZHAN \\ Department of Oncology, Changhai Hospital Affiliated to Naval Military Medical University, Shanghai 200081, P.R. China
}

Received September 23, 2020; Accepted January 28, 2021

DOI: $10.3892 /$ etm.2021.9771

\begin{abstract}
The chimeric antigen receptor (CAR) is an artificially modified fusion protein consisting of an extracellular antigen-binding domain, transmembrane domain and intracellular signalling domain. CAR-T therapy has demonstrated remarkable clinical efficacy in hematological malignancies. However, cytokine release syndrome and other side effects have hindered its application in solid tumors. CAR-natural killer (NK) cells have attracted broad attention due to their safety in clinical applications, their mechanism in recognising cancer cells and the abundance of its clinical specimens. Preclinical and clinical trials of human primary NK cells and NK-92 cell lines demonstrated that CAR-NK cells are able to fight haematological malignancies and solid tumors. However, the implication of CAR-NK cell therapy also has certain challenges, including the expansion and activation of primary NK cells in vitro, selection of CAR targets, survival time of CAR-NK cells in vivo, storage and transportation of NK cells, and efficiency of NK cell transduction. This review focuses on the latest progress of CAR-NK cells in the treatment of solid tumors.
\end{abstract}

\section{Contents}

1. Introduction

2. Advantages of CAR-natural killer (NK) cells and source of NK cells

Correspondence to: Professor Xianbao Zhan, Department of Oncology, Changhai Hospital affiliated to Naval Military Medical University, 15 Dongjiangwan Road, Hongkou, Shanghai 200081, P.R. China

E-mail: zhanxianbaoch@163.com

${ }^{*}$ Contributed equally

Abbreviations: NK, natural killer; CAR, chimeric antigen receptor; ADCC, antibody-dependent cellular cytotoxicity; HLA, human leukocyte antigen; IFN- $\gamma$, interferon- $\gamma$; KIR, killer cell immunoglobulin receptor; PD-1, programmed death 1; PD-L1, programmed death ligand 1 ; TGF- $\beta$, transforming growth factor- $\beta$; IL, interleukin

Key words: chimeric antigen receptor, natural killer cells, solid tumors, immunotherapy
3. Current status of CAR-NK cells in the treatment of the solid tumors

4. Challenges of CAR-NK cells in solid tumor treatment

5. Summary and prospect

\section{Introduction}

Natural killer (NK) celfls are a group of innate lymphocytes with a potent ability to kill virus-infected and tumor cells (1). NK cells are derived from the lymphoid stem cells in the bone marrow, differentiate and develop in the microenvironment of the bone marrow or thymus, and are mainly distributed in the peripheral blood and spleen, accounting for $\sim 10-20 \%$ of peripheral blood lymphocytes. Unlike $\mathrm{T}$ and B lymphocytes, NK cells are the third type of lymphocyte that lack specific antigen recognition receptors (2). Human $\mathrm{TCR}^{-} \mathrm{mlg}-\mathrm{CD}^{-} 6^{+} \mathrm{CD} 16^{+}$lymphocytes are defined as NK cells. According to the CD56 expression level, NK cells are divided into two subpopulations: CD56 ${ }^{\mathrm{dim}}$ and CD56 $6^{\text {bright }}$ (3). Mainly concentrated in secondary lymphoid and non-lymphoid tissues, CD56 ${ }^{\text {bright }}$ NK cells account for $\sim 10 \%$ of the total number of NK cells, regulating the immune system by secreting a large number of cytokines, including interferon (IFN)- $\gamma$, interleukin (IL)-12, IL-15 and IL-18. Specifically, IFN- $\gamma$ may induce not only dendritic cell differentiation and maturation but also $\mathrm{CD}^{+} \mathrm{T}$ cell differentiation into cytotoxic $\mathrm{T}$ cells and $\mathrm{CD} 4^{+} \mathrm{T}$ differentiation into Th cells. CD56 $6^{\mathrm{dim}} \mathrm{NK}$ cells account for $\sim 90 \%$ of the total NK cells and express a high level of FC $\gamma$ III receptors, which mediate antibody-dependent cell-mediated cytotoxicity (ADCC) and directly kill tumor cells $(2,4)$. NK cells may mediate their activity without previous sensitisation or human leukocyte antigen (HLA) matching, serving a pivotal role in immune surveillance by targeting tumor cells or virus-infected cells whose HLA-I molecules are minimally expressed $(5,6)$. NK cells may directly kill target cells and mobilise the whole immune system by producing a large number of cytokines (7). When NK cells encounter target cells, they destroy the cell membrane and dissolve the targeted cells by secreting perforin and releasing granzyme $(8,9)$. In addition, activated NK cells kill tumor cells by secreting TNF- $\alpha$, IFN- $\gamma$ and other cytokines, which directly act on target cells or attack them by activating other immune cells (10).

NK cell receptors may be divided into inhibitory and activating receptors according to their functions. The inhibitory 
NK cell receptors include the killer cell immunoglobulin-like receptors family (KIR2DL1-5, KIR3DL1-3), killer cell lectin-like receptor family (NKG2A) and LIR-1. Activated receptors include natural cytotoxic receptor family members (NKp30, NKp44 and NKp46), C-type lectin family receptors (NKG2D), activated killer immunoglobulin receptors (KIR2DS1, KIR2DS4 and KIR2DL4), FC $\gamma$ RIII (CD16) and costimulatory receptor DNAM-1 (CD226) $(11,12)$. Physiologically, NK cell inhibitory receptors recognise MHC-I molecules on the surface of body tissue cells, initiating inhibitory signal transduction and inhibiting the function of activated receptors, so that NK cells do not kill normal tissue cells (13). Pathologically, MHC-I molecules on the surface of tumor cells are downregulated or even absent, while the activated receptors are upregulated; therefore, NK cells secrete several cytokines and display cytotoxic activity (14). The activity of NK cells is regulated by various regulatory receptors on its surface. When encountering tumor cells, NK cells induce tumor cell apoptosis through different ways, including the perforin/granzyme pathway, Fas/FasL pathway and TNF- $\alpha$ /TNFR-1 pathway (9).

\section{Advantages of chimeric antigen receptor (CAR)-NK cells and source of NK cells}

Advantages of CAR-NK cells. The CAR is a fusion protein consisting of an extracellular antigen-binding domain, transmembrane domain and intracellular signalling domain. The extracellular antigen-binding domain is usually a single-chain variable fragment $(\mathrm{scFv})$, which may recognise specific antigens on tumor cells. Intracellular signalling domains, including CD28, 4-1BB (CD137) and OX40, are often designed to promote the activation and cytotoxicity of T cells. CARs may improve the specificity of autologous $\mathrm{T}$ cells for lymphoid malignancies, which has demonstrated remarkable clinical efficacy (15-17). Although autologous CAR-modified T cells exhibit antitumor activity, its manufacture is complex and expensive. In a number of patients, treatment with CAR-T cells has been associated with substantial toxic effects, including cytokine release syndrome and neurotoxicity, which involve treatment in specialised care units (18-20). There is increasing evidence on PD-1 expression in small subsets of NK cells in patients with cancer. Compared with T cells, NK cells express lower PD-1 levels and promote dendritic cell migration; therefore, CAR-NKs cells are more effective in killing solid tumor CAR-T cells. Compared with CAR-T immunotherapy, CAR-NK cells have numerous advantages. To begin with, allogeneic NK cells may be safely used as effective cells without causing graft-versus-host disease (GvHD) because they mediate non-MHC-restricted killing of tumor cells (21). Additionally, ex vivo expanded primary human NK cells produce a different spectrum of cytokines to $\mathrm{T}$ cells, including IFN- $\gamma$, IL-3 and granulocyte-macrophage colony-stimulating factor, and CAR-NK cells are associated with a lower risk of CRS $(22,23)$.

Source of CAR-NK cells. NK cells from autologous or allogeneic peripheral blood mononuclear cells are usually used for adoptive transfer of NK cells. Cord blood (CB) is a readily available allogeneic NK cell source and an 'off-the-shelf' frozen product, which provides an attractive and allogeneic NK cell for immunotherapy. Liu et al (24) demonstrated the effective cytotoxicity of CAR-CB-NK cells fixed with retroviral vectors containing CAR-CD19, IL-15 and inducible caspase-9 suicide gene to the CD19 positive cell line and primary leukaemia cells in vitro. It may significantly prolong survival time in a xenogeneic Raji lymphoma mouse model in vivo. Induced pluripotent stem cell (iPSC)-induced NK cells possess the advantages of NK-92 cell lines and primary NK cells. Researchers may obtain several NK cells with the same properties, expressing NKp44, NKp46, NKG2D and other activated receptors, which have promising prospects for development and application. Li et al (25) used human iPSCs to produce NK cells with novel CARs, which targeted cancer cells in an antigen-specific manner and improved the survival rate of xenotransplantation models of ovarian cancer. Apart from donor-derived NK cells, the NK cell line is also an important source for CAR-NK cell therapy. NK-92 cells lack almost all inhibitory KIRs and leukocyte function-related antigen-1 (LFA-1) and present higher cytotoxicity than primary NK cells (26). Preclinical and clinical studies have demonstrated the efficacy and safety of NK-92 cell line infusion in patients with cancer (27). A recent study demonstrated that KHYG-1 is a promising NK cell line for CAR-NK treatment (28).

\section{Current status of CAR-NK cells in the treatment of solid tumors}

Preclinical study. Preclinical studies of CAR-NK cells demonstrated good efficacy in patients with colorectal cancer (29), ovarian cancer (24), glioblastoma $(28,30)$, breast cancer (31), neuroblastoma (32) and other solid tumors.

NKG2D-positive colorectal cancer, ovarian cancer, prostate cancer and rhabdomyosarcoma. NKG2D ligands are upregulated in the majority of cancer cells. Chang et al (33) constructed a CAR containing NKG2D transmembrane domain, DAP10 costimulatory domain and $\mathrm{CD} 3 \zeta$ signal domain. This design enhanced the expression of NKG2D and amplified the downstream activation signal. NKG2D-DAP10-CD3 $\xi-N K$ cells presented significant cytotoxicity in prostate cancer and rhabdomyosarcoma cells. Furthermore, in the osteosarcoma mouse model, NK cells expressing NKG2D-DAP10-CD3 $\zeta$ exhibited strong antitumor activity.

Li et al (25) identified a CAR containing NKG2D transmembrane domain, 2B4 co-stimulatory domain and $\mathrm{CD} 3 \zeta$ signal domain, which mediate strong antigen-specific NK cell signalling. NK cells (NK-CAR-iPSC-NK cells) extracted from human iPSCs expressing this type of CAR had a typical NK cell phenotype and stronger antitumor activity than NK cells extracted from T-CAR-expressing iPSC (T-CAR-iPSC-NK cells) and non-CAR-expressing cells. NK-CAR-iPSC-NK cells significantly inhibited tumor growth and prolonged overall survival (OS) in an ovarian cancer xenotransplantation model. This is the first time that human iPSCs have been engineered at the stem cell level to produce iPSC-derived NK cells that express CARs. Further research on this technology may provide standardised and targeted 'off-the-shelf' lymphocytes for anticancer immunotherapy. 
Xiao et al (29) constructed a CAR by fusing the extracellular domain of natural killer cell receptor NKG2D with DAP12. Although electroporation may result in lower toxicities, the expression of NKG2D RNA-CAR significantly enhanced the cytotoxicity of NK cells on several solid tumor cell lines in vitro and had a significant therapeutic effect on mice with solid tumors. Local injection of CAR-NK cells exhibited good clinical efficacy in three patients with metastatic colorectal cancer. The results demonstrated the potential of NK cells modified with RNA-CAR in the treatment of metastatic colorectal cancer. In previous studies, NK cells expressing NKG2D CARs were usually constructed with viral vectors. When applied clinically, the DNA CAR expression mediated by integrating viral vectors cannot be easily inhibited when severe toxicity is associated with cytokine storm or on-target and off-target tumors. Using short-lived CAR-expressing cells modified with this non-integrating method, the duration and potency of CAR effects may be controlled by different dosing and infusion schemes, which may improve the safety of this CAR-NK regimen. Electroporation may result in lower toxicities, but different dosing and infusion schemes may result in immune rejection (29).

EGFRvIII scFv-positive glioblastoma. Murakami et al (28) constructed a lentivirus vector composed of the transmembrane domain of EGFR variant III (EGFRvIII), CD3 $\zeta$ signal domain, CD137 (4-1BB) and CD28 costimulatory domain. These lentivirus vectors were introduced into the human NK cell line KHYG-1. It was found that CAR-KHYG-1 cells had strong antigen specificity and cytotoxicity to U87 cells stably expressing EGFRvIII in vitro. As an 'off-the-shelf' treatment method, continuous expansion of CAR-KHYG-1 cells may have a clinical effect in cancer patients. EvCAR-KHYG-1 may be an effective treatment for glioblastoma. Müller et al (30) reported that NK cells that overexpressed CXCR4 EGFRvIII-specific CAR promoted immunotherapy of glioblastoma that secretes XCL12/SDF-1 $\alpha$. The engineered NK cells expressing chemokine receptor and tumor-specific CAR are a promising tool for improving adoptive immunotherapy for tumors.

EpCAM-positive breast cancer. A preclinical study (31) demonstrated that NK cells co-expressing CAR and IL-15 continued to proliferate even in the absence of exogenous cytokines and exhibited highly selective cytotoxicity against breast cancer cells expressing EpCAM, which were resistant to the cytotoxicity of unmodified NK cells.

GD2-positive neuroblastoma, melanoma and breast cancer. Esser et al (32) constructed a clone derivative of the NK-92 cell line, which stably expressed GD2-specific CAR. The GD2-CAR-NK92 cells demonstrated strong cytotoxicity against neuroblastoma cell lines, which depended on the specific recognition of target antigens. The GD2-specific NK cells exhibited cytotoxicity against melanoma cells, breast cancer cells and glioblastoma cells that express GD2, indicating the potential clinical application value of GD2-CAR-NK92 cells.

Clinical trial. To date, 10 clinical trials utilising CAR-expressing NK cells for the treatment of solid tumors (NCT02839954,
NCT03692637, NCT03692663, NCT03415100, NCT03940820, NCT03940833, NCT03941457, NCT03931720, NCT03656705 and NCT03383978) have been initiated (Table I).

The first study (clinical trial no. NCT02839954, https://www.clinicaltrials.gov/ct2/show/NCT02839954) was initiated by researchers from the First People's Hospital of Hefei to investigate the safety and efficacy of CAR-pNK cell immunotherapy for MUC1-positive recurrent or refractory solid tumors. The enrolled patients received CAR-pNK cell immunotherapy with specific CAR targeting the MUC1 antigen. The main inclusion criteria were as follows: MUC1-positive tumors that lack available treatment methods and have a poor prognosis. Currently available treatments were included. Eligible MUC1-positive tumors in the clinical study included brain malignant glioma, colorectal cancer, gastric cancer, hepatocellular carcinoma, non-small cell lung cancer, pancreatic cancer and triple-negative basal breast cancer. MUC1 expression in tumor tissues was confirmed by immunohistochemistry. CD27 and 4-1BB (CD137) are included as signal domains of CAR in the present study. All patients were followed up until August 2017. A total of 10 patients with non-small cell lung cancer, pancreatic cancer, liver cancer, colon cancer and ovarian cancer were enrolled. Serious side effects, including cytokine release syndrome or myelosuppression, were not observed in these 10 patients, indicating that the treatment was safe and tolerable. However, there is no further report about this research including efficacy data.

\section{Challenges of CAR-NK cells in solid tumor treatment}

Although CAR-NK cells exhibit low cytotoxicity and GvHD risk, certain challenges remain, including expansion and activation of NK cells, CAR target selection, cell survival time of CAR-NK in vivo, transduction method, and storage and transportation of NK cell products.

Expansion and activation of primary NK cells. The expansion of primary NK cells in vitro is the primary challenge of CAR-NK cell therapy. Since the number of NK cells from a single donor is far from enough for the treatment, the expansion and activation of NK cells are important (34). Interleukin may be used to expand and activate NK cells. The process of amplifying NK cells by adding IL-2 or IL-15 alone usually requires 2-3 weeks (35). The combination of IL-2 and IL-21 is better than IL-2 alone in promoting NK cells to curb tumor cells $(36,37)$. Irradiated K562 cells may be used as donor cells during the expansion of primary NK cells; however, they must be completely removed prior to infusion as K562 is a cancer cell line (38).

CAR targets election. The CAR structure is important in the construction of CAR-NK cells. An extracellular binding domain is the core component of CAR, which determines the specificity of effective cells. Presently, there is no specific antigen that is exclusively expressed on tumor cells; instead, tumor cells express several tumor-related antigens. Therefore, CAR structures with several antigen receptor complexes may be designed to recognise tumor-related antigen combinations to improve the specificity and versatility of NK cells. Activated or inhibited receptors may be used in the construction of 
Table I. Clinical trials using CAR-natural killer cells for treatment of solid tumors.

\begin{tabular}{lllll}
\hline NCT number & Intervention & \multicolumn{1}{c}{ Condition/disease } & Phase & Recruitment status \\
\hline NCT02839954 & MUC1 & $\begin{array}{l}\text { Hepatocellular carcinoma; non-small cell } \\
\text { lung cancer; pancreatic carcinoma; } \\
\text { triple-negative invasive breast carcinoma; } \\
\text { malignant glioma of the brain; colorectal } \\
\text { carcinoma; gastric carcinoma }\end{array}$ & $\begin{array}{l}\text { Phase 1 } \\
\text { Phase 2 }\end{array}$ & Unknown \\
& & Epithelial ovarian cancer & Early phase 1 & Not yet recruiting \\
NCT03692637 & Mesothelin & Castration-resistant prostate cancer & Early phase 1 & Not yet recruiting \\
NCT03692663 & PSMA & Solid tumors & Phase 1 & Unknown \\
NCT03415100 & NKG2D ligands & Phase 1 & Recruiting \\
NCT03940820 & ROBO1 & Solid tumors & Phase 2 & Phase 1 \\
NCT03940833 & BCMA & Multiple myeloma & Phase 2 & Recruiting \\
NCT03941457 & ROBO1 & Pancreatic cancer & Phase 1 & Recruiting \\
NCT03931720 & ROBO1 & Malignant tumors & Phase 2 & Phase 1 \\
NCT03656705 & CCCR & PK-92/5.28.z & Gon-small cell lung cancer & Recruiting \\
NCT03383978 & Glioblastoma & Phase 1 & Phase 1 & Enrolling by invitation \\
\hline
\end{tabular}

Data was obtained from http://clinicaltrials.gov.

CAR. NKG2D is an important activated receptor in NK cells. Inhibition of MICA and MICB may promote the antitumor immunity of NK cells mediated by NKG2D and CD16 Fc receptors $(39,40)$. CD244 $(2 \mathrm{~B} 4)$ is a signal domain of the lymphocyte-activating molecule-related receptor, which regulates the effective stimulation and co-stimulation signals in NK cells, and may promote signal transduction in NK cells that are relocated to tumor cells (41).

Persistence of CAR-NK cells in vivo. Non-engineered NK cells usually disappear 2 weeks after infusion, which limits the clinical application of NK cells $(42,43)$. Liu et al $(44)$ reported that infused CAR-NK cells lasted for at least 12 months at a low level in vivo. The long-term persistence of HLA-mismatched CAR-NK cells may be mediated by the loose environment created by lymphocyte depletion therapy and ectopic expression of IL-15 by CAR-NK cells, which enhanced the expansion and persistence of transformed NK cells. However, these persistent cells were insufficient to prevent recurrence, which may limit the application of CAR-NK cells in tumors. IL-15 may be used to enhance the persistence of CAR-NK cells in the future.

Transduction pathway of CAR-NK cells. CAR can be transferred into NK cells through viral and nonviral vectors. Viral vectors are widely used in CAR-NK cell therapy as they can be stably integrated into the genome. Retroviral vectors have high transfection efficiency but have certain disadvantages, including high risk and cost, limited viral vector capacity, insertion mutation, carcinogenesis and induction of immune response $(45,46)$. Lentivirus vectors have a moderate number of insertion mutations, although the lentivirus transduction efficiency in peripheral blood-derived NK cells is relatively low $(8-16 \%)$ (47). The transfection efficiency of viral vectors is extremely high, but there are certain obvious defects; therefore, it is imperative to investigate and optimise nonviral vectors. The Sleeping Beauty (SB) transposon vector provides a safe, effective and economic method to integrate genetic information through a gene transfer vector, which may overcome the shortcomings of viral vectors. However, the applicability of the SB transposon system in transferring CAR into primary NK cells remains to be verified (48-50). It is considered a safe and economic method to transfect CAR-NK cells with mRNA, which demonstrated significant cytotoxicity in xenotransplantation cancer models $(33,51)$.

Storage and transportation of NK cell products. NK cells are extremely sensitive to freezing and thawing when activated by cytokines, and the cytotoxicity of NK cells is significantly decreased following thawing $(52,53)$. The sensitivity of NK cells to cryopreservation makes it difficult to store and transport NK cells, which is the limitation of CAR-NK cell therapy. Adequate cell density is also important in the transportation process. High NK cell levels will lead to cell inactivity. As a result of the temperature sensitivity of NK cells, they need to be transported at body temperature to maintain the cytotoxicity.

\section{Summary and prospect}

Cancer immunotherapy based on adoptive cell therapy or immune-checkpoint inhibition has revolutionised cancer care. Current ICB therapies require tumor neoantigen presentation through MHC-I to elicit antitumor immunity. A low response rate, acquired resistance and severe side effects 
have limited the clinical outcomes of immune checkpoint therapy (immunisation with mannosylated nanovaccines and inhibition of the immune-suppressing microenvironment sensitise melanoma to immune checkpoint modulators).

Anti-CD19 CAR-T cells have ushered into a new era of treatment for acute lymphoblastic leukaemia (ALL). Recently, basic and clinical studies on CAR-T cell therapy for multiple solid tumors have been published, with some demonstrating encouraging progress. However, CAR-T therapy continues to have numerous challenges. Physical barriers of tumor stroma and immune cells make it difficult for CAR-T cells to infiltrate into solid tumors. Additionally, the heterogeneity of tumor target antigen and mild specificity of CAR T cells to tumor cells hindered its antitumor efficacy.

Genetically-engineered CAR human macrophages (CAR macrophages, CAR-Ms) demonstrated antigen-specific phagocytosis and tumor clearance in vitro, imparting a sustained pro-inflammatory (M1) phenotype and converting bystander M2 macrophages to M1. A single infusion of human CAR-Ms decreased tumor burden and prolonged overall survival (OS) in solid tumor xenograft mouse models. However, the demanding culture conditions and safety concerns indicated that there is still a long way to go before it can be tested and utilised in clinical trials (human CAR macrophages for cancer immunotherapy).

NK cells may mediate their activity without previous sensitisation or human leukocyte antigen (HLA) matching, serving a pivotal role in immune surveillance by targeting tumor cells or virus-infected cells in HLA-I molecules that are expressed at a low level $(5,6)$. Although CAR-NK cell therapy has been proven to be an effective antitumor method, its long-term clinical effect remains limited. The combination therapy based on NK cells provides a novel direction for tumor immunotherapy. The combination therapy targeting the immune environment may be conducive to CAR-NK cell therapy. The combination of activated NK cells and anti-PD-1 therapy has been demonstrated to navigate abundant immune cells into tumor tissues and proven to be safe and effective in the long term in preclinical tumor models (54). A recent study demonstrated that IFN- $\beta$ may activate the TRAIL signalling pathway and enhance NK cell-induced cytotoxicity of nasopharyngeal carcinoma (NPC) cells by upregulating TRAIL expression in NK cells. PD-1 blockade may further enhance the cytotoxicity of IFN- $\beta$ on NPC cells, suggesting that IFN- $\beta$ combined with PD-L1/PD-1 blockade may have a clinical application (55). The high expression of the corresponding ligands of NK cell inhibitory receptors on the surface of tumor cells is the key factor limiting the function of CAR-NK cells. Therefore, silencing NK cell inhibitory receptors and ligands may be conducive to improving the efficiency of CAR-NK cells (56). Furthermore, chemotherapy may also be combined with CAR-NK cells in the treatment of tumors, as chemotherapy can not only eliminate resident cell groups but also induce genotoxic stress and enhance the sensitivity of tumor cells to NK cells $(57,58)$. Furthermore, the safety and clinical effect of the treatment of stage III/IV pancreatic cancer with the combination of percutaneous irreversible electroporation and allogeneic NK cells was demonstrated. The results suggested that combination therapy may significantly improve the median progression-free survival and OS of patients with stage III pancreatic cancer and prolong the median OS of patients with stage IV pancreatic cancer. Multiple infusions of allogeneic natural killer cells may improve the prognosis of stage III pancreatic cancer. As a novel antitumor cell immunotherapy method, CAR-NK cells have broad applications. Combination therapy based on CAR-NK cells provides a novel direction for immunotherapy in the future.

\section{Acknowledgements}

Not applicable.

\section{Funding}

The present study was supported by grants from the National Natural Science Foundation of China (grant no. 81672892), Changhai Hospital (grant nos. 2019YXK019 and 2020YXK029) and Shanghai Anticancer Association EYAS PROJECT (grant no. SACA-CY1C12).

\section{Availability of data and materials}

Not applicable.

\section{Authors' contributions}

$\mathrm{XP}, \mathrm{LiC}, \mathrm{LoC}$ and XZ conceived the present study, XP, BW and YW performed the literature search and data analysis. The first draft of the manuscript was written by XP, LiC and LoC, and XZ, BW, YW and XZ critically revised the work. All authors read and approved the final manuscript.

\section{Ethics approval and consent to participate}

Not applicable.

\section{Patient consent for publication}

Not applicable.

\section{Competing interests}

The authors declare that they have no competing interests.

\section{References}

1. Glimcher L, Shen FW and Cantor H: Identification of a cell-surface antigen selectively expressed on the natural killer cell. J Exp Med 145: 1-9, 1997.

2. Cooley S, Parham P and Miller JS: Strategies to activate NK cells to prevent relapse and induce remission following hematopoietic stem cell transplantation. Blood 131: 1053-1062, 2018.

3. Wu Y, Tian Z and Wei H: Developmental and functional control of natural killer cells by cytokines. Front Immunol 8: 930, 2017.

4. Crouse J, Xu HC, Lang PA and Oxenius A: NK cells regulating T cell responses: Mechanisms and outcome. Trends Immunol 36: 49-58, 2015.

5. Lanier LL: Up on the tightrope: Natural killer cell activation and inhibition. Nat Immunol 9: 495-502, 2008.

6 . Wu J and Lanier LL: Natural killer cells and cancer. Adv Cancer Res 90: 127-156, 2003.

7. Chong WP, van Panhuys N, Chen J, Silver PB, Jittayasothorn Y, Mattapallil MJ, Germain RN and Caspi RR: NK-DC crosstalk controls the autopathogenic Th17 response through an innate IFN- $\gamma$-IL-27 axis. J Exp Med 212: 1739-1752, 2015. 
8. Jung IH, Kim DH, Yoo DK, Baek SY, Jeong SH, Jung DE, Park SW and Chung YY: In vivo study of natural killer (NK) cell cytotoxicity against cholangiocarcinoma in a nude mouse model. In Vivo 32: 771-781, 2018.

9. Chester C, Fritsch K and Kohrt HE: Natural killer cell immunomodulation: Targeting activating, inhibitory, and co-stimulatory receptor signaling for cancer immunotherapy. Front Immuno 6 : $601,2015$.

10. Paul S and Lal G: The molecular mechanism of natural killer cells function and its importance in cancer immunotherapy. Front Immunol 8: 1124, 2017.

11. Valiante NM, Uhrberg M, Shilling HG, Lienert-Weidenbach K, Arnett KL, D'Andrea A, Phillips JH, Lanier LL and Parham P: Functionally and structurally distinct NK cell receptor repertoires in the peripheral blood of two human donors. Immunity 7: 739-751, 1997.

12. Konjević G, Vuletić A and Mirjačić Martinović K: Natural killer cell receptors: Alterations and therapeutic targeting in malignancies. Immunol Res 64: 25-35, 2016.

13. Yokoyama WM and Kim S: How do natural killer cells find self to achieve tolerance? Immunity 24: 249-257, 2006.

14. Carotta S: Targeting NK cells for anticancer immunotherapy: Clinical and preclinical approaches. Front Immunol 7: 152, 2016.

15. Kochenderfer JN, Dudley ME, Feldman SA, Wilson WH, Spaner DE, Maric I, Stetler-Stevenson M, Phan GQ, Hughes MS, Sherry RM, et al: B-cell depletion and remissions of malignancy along with cytokine-associated toxicity in a clinical trial of anti-CD19 chimeric-antigen-receptor transduced T cells. Blood 119: 2709-2720, 2012.

16. Porter DL, Levine BL, Kalos M, Bagg A and June CH: Chimeric antigen receptor-modified $\mathrm{T}$ cells in chronic lymphoid leukemia. N Engl J Med 365: 725-733, 2011.

17. Maude SL, Teachey DT, Porter DL and Grupp SA: CD19-targeted chimeric antigen receptor T-cell therapy for acute lymphoblastic leukemia. Blood 125: 4017-4023, 2015.

18. Lee DW, Santomasso BD, Locke FL, Ghobadi A, Turtle CJ, Brudno JN, Maus MV, Park JH, Mead E, Pavletic S, et al: ASTCT consensus grading for cytokine release syndrome and neurologic toxicity associated with immune effector cells. Biol Blood Marrow Transplant 25: 625-638, 2019.

19. Davila ML, Riviere I, Wang X, Bartido S, Park J, Curran K, Chung SS, Stefanski J, Borquez-Ojeda O, Olszewska M, et al: Efficacy and toxicity management of $19-28 z$ CAR T cell therapy in B cell acute lymphoblastic leukemia. Sci Transl Med 6: 224ra25, 2014.

20. Neelapu SS, Tummala S, Kebriaei P, Wierda W, Locke FL, Lin Y, Jain N, Daver N, Gulbis AM, Adkins S, et al: Toxicity management after chimeric antigen receptor $\mathrm{T}$ cell therapy: One size does not fit 'ALL'. Nat Rev Clin Oncol 15: 218, 2018.

21. Tang X, Yang L, Li Z, Nalin AP, Dai H, Xu T, Yin J, You F, Zhu M, Shen W, et al: First-in-man clinical trial of CAR NK-92 cells: Safety test of CD33-CAR NK-92 cells in patients with relapsed and refractory acute myeloid leukemia. Am J Cancer Res 8: 1083-1089, 2018.

22. Morgan RA, Yang JC, Kitano M, Dudley ME, Laurencot CM and Rosenberg SA: Case report of a serious adverse event following the administration of $T$ cells transduced with a chimeric antigen receptor recognizing ERBB2. Mol Ther 18: 843-851, 2010.

23. Shaffer BC, Le Luduec JB, Forlenza C, Jakubowski AA, Perales MA, Young JW and Hsu KC: Phase II study of haploidentical natural killer cell infusion for treatment of relapsed or persistent myeloid malignancies following allogeneic hematopoietic cell transplantation. Biol Blood Marrow Transplant 22: 705-709, 2016.

24. Liu E, Tong Y, Dotti G, Shaim H, Savoldo B, Mukherjee M, Orange J, Wan X, Lu X, Reynolds A, et al: Cord blood NK cells engineered to express IL-15 and a CD19-targeted CAR show long-term persistence and potent antitumor activity. Leukemia 32: 520-531, 2018

25. Li Y, Hermanson DL, Moriarity BS and Kaufman DS: Human iPSC-derived natural killer cells engineered with chimeric antigen receptors enhance anti-tumor activity. Cell Stem Cell 23: 181-192.e5, 2018.

26. Maki G, Klingemann HG, Martinson JA and Tam YK: Factors regulating the cytotoxic activity of the human natural killer cell line, NK-92. J Hematother Stem Cell Res 10: 369-383, 2001.

27. Suck G, Odendahl M, Nowakowska P, Seidl C, Wels WS, Klingemann HG and Tonn T: NK-92: An 'off-the-shelf therapeutic' for adoptive natural killer cell-based cancer immunotherapy. Cancer Immunol Immunother 65: 485-492, 2016.
28. Murakami T,NakazawaT,Natsume A,NishimuraF, Nakamura M, Matsuda R, Omoto K, Tanaka Y, Shida Y, Park YS, et al: Novel human NK cell line carrying CAR targeting EGFRvIII induces antitumor effects in glioblastoma cells. Anticancer Res 38: 5049-5056, 2018.

29. Xiao L, Cen D, Gan H, Sun Y, Huang N, Xiong H, Jin Q, Su L, Liu X, Wang K, et al: Adoptive transfer of NKG2D CAR mRNA-engineered natural killer cells in colorectal cancer patients. Mol Ther 27: 1114-1125, 2019.

30. Müller N, Michen S, Tietze S, Töpfer K, Schulte A, Lamszus K, Schmitz M, Schackert G, Pastan I and Temme A: Engineering NK cells modified with an EGFRvIII-specific chimeric antigen receptor to overexpress CXCR4 improves immunotherapy of CXCL12/SDF-1 $\alpha$-secreting glioblastoma. J Immunother 38: 197-210, 2015.

31. Sahm C, Schönfeld K and Wels WS: Expression of IL-15 in NK cells results in rapid enrichment and selective cytotoxicity of gene-modified effectors that carry a tumor-specific antigen receptor. Cancer Immunol Immunother 61: 1451-1461, 2012.

32. Esser R, Müller T, Stefes D, Kloess S, Seidel D, Gillies SD, Aperlo-Iffland C, Huston JS, Uherek C, Schönfeld K, et al: NK cells engineered to express a GD2-specific antigen receptor display built-in ADCC-like activity against tumour cells of neuroectodermal origin. J Cell Mol Med 16: 569-581, 2012.

33. Chang YH, Connolly J, Shimasaki N, Mimura K, Kono K and Campana D: A chimeric receptor with NKG2D specificity enhances natural killer cell activation and killing of tumor cells. Cancer Res 73: 1777-1786, 2013.

34. Becker PS, Suck G, Nowakowska P, Ullrich E, Seifried E, Bader P, Tonn T and Seidl C: Selection and expansion of natural killer cells for NK cell-based immunotherapy. Cancer Immunol Immunother 65: 477-484, 2016.

35. Li Y, Liu B, Ding S, Liu C, Chen T, Li L, Shao Z and Fu R: Availability of NK cell expansion agent combined with recombinant IL-2 and IL-15 stimulation on the expansion and high-purity of NK cells in patients with immune-related pancytopenia in vitro. Mol Med Rep 20: 4358-4366, 2019.

36. Ojo EO, Sharma AA, Liu R, Moreton S, Checkley-Luttge MA, Gupta K, Lee G, Lee DA, Otegbeye F, Sekaly RP, et al: Membrane bound IL-21 based NK cell feeder cells drive robust expansion and metabolic activation of NK cells. Sci Rep 9: 14916, 2019.

37. Kweon S, Phan MT, Chun S, Yu H, Kim J, Kim S, Lee J, Ali AK, Lee SH, Kim SK, et al: Expansion of human NK cells using K562 cells expressing OX40 ligand and short exposure to IL-21. Front Immunol 10: 879, 2019.

38. Phan MT, Lee SH, Kim SK and Cho D: Expansion of NK cells using genetically engineered K562 feeder cells. Methods Mol Biol 1441: 167-174, 2016.

39. Ferrari de Andrade L, Tay RE, Pan D, Luoma AM, Ito Y, Badrinath S, Tsoucas D, Franz B, May KF Jr, Harvey CJ, et al: Antibody-mediated inhibition of MICA and MICB shedding promotes NK cell-driven tumor immunity. Science 359: $1537-1542,2018$.

40. Liu H, Wang S, Xin J, Wang J, Yao C and Zhang Z: Role of NKG2D and its ligands in cancer immunotherapy. Am J Cancer Res 9: 2064-2078, 2019.

41. Pende D, Meazza R, Marcenaro S, Aricò M and Bottino C: 2B4 dysfunction in XLP1 NK cells: More than inability to control EBV infection. Clin Immunol 204: 31-36, 2019.

42. Miller JS, Soignier Y, Panoskaltsis-Mortari A, McNearney SA, Yun GH, Fautsch SK, McKenna D, Le C, Defor TE, Burns LJ, et al: Successful adoptive transfer and in vivo expansion of human haploidentical NK cells in patients with cancer. Blood 105: 3051-3057, 2005.

43. Dunai C and Murphy WJ: NK cells for PD-1/PD-L1 blockade immunotherapy: Pinning down the NK cell. J Clin Invest 128: 4251-4253, 2018

44. Liu E, Marin D, Banerjee P, Macapinlac HA, Thompson P, Basar R, Nassif Kerbauy L, Overman B, Thall P, Kaplan M, et al: Use of CAR-transduced natural killer cells in CD19 positive lymphoid tumors. N Engl J Med 382: 545-553, 2020.

45. Wang GP, Garrigue A, Ciuffi A, Ronen K, Leipzig J, Berry C, Lagresle-Peyrou C, Benjelloun F, Hacein-Bey-Abina S, Fischer A, et al: DNA bar coding and pyrosequencing to analyze adverse events in therapeutic gene transfer. Nucleic Acids Res 36: e49, 2008.

46. Hu WS and Pathak VK: Design of retroviral vectors and helper cells for gene therapy. Pharmacol Rev 52: 493-511, 2000.

47. Hu Y, Tian ZG and Zhang C: Chimeric antigen receptor (CAR)-transduced natural killer cells in tumor immunotherapy. Acta Pharmacol Sin 39: 167-176, 2018. 
48. Chen X, Xu D, Zhang J and Tang J: Future perspectives of sleeping beauty transposon system in cancer research. EBioMedicine 46: 2-3, 2019.

49. Chicaybam L, Abdo L, Carneiro M, Peixoto B, Viegas M, de Sousa P, Fornazin MC, Spago MC, Albertoni Laranjeira AB, de Campos-Lima PO, et al: CAR T cells generated using sleeping beauty transposon vectors and expanded with an EBV-transformed lymphoblastoid cell line display antitumor activity in vitro and in vivo. Hum Gene Ther 30: 511-522, 2019.

50. Hou X, Du Y, Deng Y, Wu J and Cao G: Sleeping Beauty transposon system for genetic etiological research and gene therapy of cancers. Cancer Biol Ther 16: 8-16, 2015.

51. Ng YY, Tay JCK and Wang S: CXCR1 expression to improve anti-cancer efficacy of intravenously injected CAR-NK cells in mice with peritoneal xenografts. Mol Ther Oncolytics 16: 75-85, 2019.

52. Fang F, Xiao W and Tian Z: NK cell-based immunotherapy for cancer. Semin Immunol 31: 37-54, 2017.

53. Lapteva N, Durett AG, Sun J, Rollins LA, Huye LL, Fang J, Dandekar V, Mei Z, Jackson K, Vera J, et al: Large-scale ex vivo expansion and characterization of natural killer cells for clinical applications. Cytotherapy 14: 1131-1143, 2012.

54. Shevtsov M, Pitkin E, Ischenko A, Stangl S, Khachatryan W, Galibin O, Edmond S, Lobinger D and Multhoff G: Ex vivo Hsp70-activated NK cells in combination with PD-1 inhibition significantly increase overall survival in preclinical models of glioblastoma and lung cancer. Front Immunol 10: 454, 2019.
55. Makowska A, Braunschweig T, Denecke B, Shen L, Baloche V, Busson $\mathrm{P}$ and Kontny $\mathrm{U}$ : Interferon $\beta$ and anti-PD-1/PD-L1 checkpoint blockade cooperate in NK cell-mediated killing of nasopharyngeal carcinoma cells. Transl Oncol 12: 1237-1256, 2019.

56. Perez-Santos M, Guerrero-González T, Gómez-Conde E, Cebada J, Flores A and Villa-Ruano N: Treatment of cancer with an anti-KIR antibody: A patent evaluation of US9879082 and US2018208652. Expert Opin Ther Pat 30: 159-162, 2020.

57. Zhang X, Wang D, Li Z, Jiao D, Jin L, Cong J, Zheng X and Xu L: Low-dose gemcitabine treatment enhances immunogenicity and natural killer cell-driven tumor immunity in lung cancer. Front Immunol 11: 331, 2020.

58. Issels RD, Lindner LH, von Bergwelt-Baildon M, Lang P, Rischpler C, Diem H, Mosetter B, Eckl J, Schendel DJ, Salat C, et al: Systemic antitumor effect by regional hyperthermia combined with low-dose chemotherapy and immunologic correlates in an adolescent patient with rhabdomyosarcoma-a case report. Int J Hyperthermia 37: 55-65, 2020.

This work is licensed under a Creative Commons Attribution-NonCommercial-NoDerivatives 4.0 International (CC BY-NC-ND 4.0) License. 